\title{
Prognostic significance of CD95, P53, and BCL2 expression in extranodal non-Hodgkin's lymphoma
}

\author{
Anastasios Chatzitolios • Ioannis Venizelos • \\ Gregory Tripsiannis • George Anastassopoulos • \\ Nikolaos Papadopoulos
}

Received: 27 April 2009/Accepted: 15 March 2010/Published online: 30 March 2010

(C) Springer-Verlag 2010

\begin{abstract}
Apoptosis-related proteins play an important role in lymphoma cell death during chemotherapy. In our study, we investigated the prognostic significance of CD95, BCL2, and P53 expression in extranodal non-Hodgkin's lymphoma (NHL). We examined 71 patients with extranodal NHL [45 diffuse large B-cell lymphomas (DLBCLs) and 26 mucosa-associated lymphoid tissue lymphomas (MALTLs)], 35 male and 36 female, with a median age of 65.8 years. The most common site of origin was the stomach $(N=31)$. Paraffin-embedded specimens were analyzed immunohistochemically for CD95, BCL2, and P53 expression. Multivariate analysis revealed that in DLBCLs, positive CD95 and negative BCL2 expression were independent prognostic factors for overall survival. We reached the same conclusion for MALTLs, with positive CD95 and negative P53 expression. In DLBCLs, the 5-year overall survival rate was $71.5 \%$ for the $\mathrm{CD} 95$-positive cases and $35 \%$ for the CD95-negative cases $(p=0.004)$ and the 5 year overall survival was significantly better in BCL2negative cases $(70.8 \%)$ when compared to BCL2-positive cases $(37 \% ; p=0.009)$. In MALTLs, the 5 -year overall survival rate for the CD95-positive and CD95-negative groups was $89.5 \%$ and $42.9 \%$, respectively $(p=0.004)$ and the 5-year overall survival rate was $50 \%$ for the P53-
\end{abstract}

A. Chatzitolios $(\bowtie) \cdot$ I. Venizelos

Pathology Department, Ippokration Hospital,

5 Zagoras st.,

54636 Thessaloniki, Greece

e-mail: axtolios@yahoo.gr

G. Tripsiannis $\cdot$ G. Anastassopoulos $\cdot$ N. Papadopoulos

Department of Histology-Embryology,

Democritus University of Thrace,

Alexandroupolis, Greece positive cases and $88.9 \%$ for the P53-negative cases ( $p=$ 0.016). In conclusion, positive CD95 expression proved to be a good prognostic factor of overall survival in both extranodal DLBCLs and MALTLs. In contrast, positive expression of BCL2 and P53 was found to be unfavorably associated with survival in extranodal DLBCLs and MALTLs, respectively.

Keywords CD95 · P53 - BCL2 - Prognostic significance · DLBCL $\cdot$ MALTL $\cdot$ Extranodal non-Hodgkin's lymphoma

\section{Introduction}

Extranodal non-Hodgkin's lymphomas are defined as such, when the major tumor mass is located at an extranodal site. These lymphomas can arise in all organs, but most commonly in the gastrointestinal tract [1]. Extranodal lymphomas heterogeneous present with significant variations in relation to prognosis and treatment response [1] and relatively few studies have focused on identifying tissue expression of biological markers that could predict prognosis for a given patient.

CD95 (FAS/APO-1), an important regulator of apoptosis, is a membrane glycoprotein belonging to the tumor necrosis factor/nerve growth factor receptor family, which can trigger apoptosis in some lymphoid cell lines. Incubation of CD95 positive, activated $\mathrm{T}$ and $\mathrm{B}$ lymphocytes, and natural killer cells with antiCD95 monoclonal antibodies has been shown to induce apoptotic cell death. The mere presence of CD95 on the cell surface, however, is insufficient for the delivery of the apoptotic signal, since lymphocyte activation is a necessary prerequisite to enable the cell to receive the apoptotic signal transduced by CD95 [2-5]. 
P53 protein plays a vital role in suppressing the development of cancer. The accumulation of P53 protein in response to DNA damage is a well-established event and appears to induce growth arrest and apoptosis by transcriptional regulation of other genes. The P53 gene is located on chromosome $17 \mathrm{p}$, a frequent site of allelic loss in many tumors. Mutations of the P53 gene may represent the commonest genetic event in human malignancy. It has been reported that a high proportion of breast and colon carcinomas show positive immunostaining for P53 protein. Meanwhile, aberrant expression of P53 is regarded as an important anti-apoptotic factor in the progression of B-cell lymphoma [6-8].

BCL2 oncoprotein plays a central role in apoptosis, acting as an inhibitor of the apoptotic process. It belongs to a family of proteins engaged in the promotion/inhibition of apoptosis. For example, BCL-XL has an anti-apoptotic function, whereas BAX, BAK, BCL-XS, BIK, and BID display pro-apoptotic function. BCL2 protein is considered to be an important contributor for multidrug resistance, because of its anti-apoptotic effects. The antibody that detects BCL2 in paraffin blocks has numerous applications in studies of apoptosis, e.g., in hematological malignancies and in other malignant diseases [6, 9-12].

The aim of our study is to investigate the relation between CD95, P53, and BCL2 expression and prognosis in patients with extranodal DLBCL and MALTL.

\section{Design and methods}

One-hundred and nineteen patients, mainly from Northern Greece, were diagnosed at the Pathology Department of Ippokration Hospital with extranodal non-Hodgkin's lymphoma, from 1997 to 2007. We were able to retrieve archival paraffin blocks of 108 histopathologic samples. Therefore, 11 patients were excluded from our study because no archival tissue was available for analysis. Also, 27 patients had been lost to follow-up. We decided to exclude from our study another ten patients with certain histological entities that could be categorized as extranodal lymphomas (mycosis fungoides, skin- associated lymphoid tissue lymphoma, diffuse T-cell rich B-cell lymphoma, and post-transplant lymphoproliferative disorder polymorphic) because the small sample size would affect statistical significance. The remaining 71 specimens, 45 DLBCLs and 26 MALTLs, were considered for the study. The patients were 35 males $(49.2 \%)$ and 36 females (50.8\%) with ages ranging from 16 to 83 years (median 65.8 years). The duration of the follow-up ranged from 5 to 111 months (median 48.96 months). The most common site of the extranodal lymphoma was the stomach $(N=31)$. The histological subtypes and sites of involvement are described analytically in Table 1.
Table 1 Extranodal non-Hodgkin's lymphomas: sites and histological subtypes

\begin{tabular}{llll}
\hline & DLBCL & MALTL & \\
\hline Stomach & 13 & 18 & 31 \\
Skin & 5 & - & 5 \\
Small intestine & 7 & 2 & 9 \\
Salivary glands & 2 & 3 & 5 \\
Liver & 1 & 1 & 2 \\
Tonsils & 2 & - & 2 \\
Nasopharynx & 2 & - & 2 \\
Breast & 1 & 1 & 2 \\
Uterus & 1 & 1 & 2 \\
Ileocecal valve & 2 & - & 2 \\
Thyroid & 1 & - & 1 \\
Larynx & 1 & - & 1 \\
Large intestine & 1 & - & 1 \\
Spleen & 1 & - & 1 \\
Kidney & 1 & - & 1 \\
Adrenal gland & 1 & - & 1 \\
Testis & 1 & - & 1 \\
Bone & 1 & - & 1 \\
Soft tissue & 1 & - & 1 \\
Total & 45 & & \\
\hline & & 26 &
\end{tabular}

Abbreviations: DLBCL (diffuse large B-cell lymphoma),

MALTL (mucosa associated lymphoid tissue lymphoma)

All specimens were reviewed and reclassified based on morphological and immunohistochemical findings according to the WHO classification (2001). All patients were staged according to the Ann Arbor staging system. The International Prognostic Index (IPI) was calculated as described in the International Non-Hodgkin's Prognostic Factors Project.

All specimens derived from formalin-fixed, paraffinembedded archival material. Paraffin blocks were sectioned at $4 \mu \mathrm{m}$ thick sections and were initially stained with hematoxylin and eosin.

Treatment

Fifteen patients had been treated with surgery and excision of bulky tumors had been followed by radiotherapy and/or chemotherapy.

All the patients with DLBCLs received the following scheme of chemotherapy: Rituximab-Cyclophosphamide/ Doxorubicin/Vincristine/Prednisone (R-CHOP), CHOP, Rituximab-Cyclophosphamide/Epirubicin/Vincristine/ Prednisone (R-CEOP) or CEOP; four cycles were given for stage I-IIA disease and six to eight cycles for stage IIB-IV disease.

Patients with gastric MALTLs associated with Helicobacter pylori infection had received eradication treatment. 
In the absence of H. pylori or when H. pylori treatment had failed, MALTLs had been treated with radiotherapy. In advanced stages or when other treatments had failed, patients with MALTLs had been treated with chemotherapy (CHOP or R-CHOP).

\section{Immunohistochemistry}

Immunohistochemical staining for CD95, P53, and BCL2 was performed on 4- $\mu$ m-thick formalin-fixed, paraffinembedded sections following deparaffinization in xylene and rehydration in a graded series of ethanols. Slides were stained using Fas (CD95) lyophilized mouse monoclonal antibody (clone LOB3-17, 1:20, Novocastra, Newcastle, UK), p53 protein, lyophilized mouse monoclonal antibody, (Clone DO-7, 1:50, Novocastra, Newcastle, UK) and bcl2 oncoprotein, lyophilized mouse monoclonal antibody, (Clone 3.1, 1:50, Novocastra, Newcastle, UK) following antigen retrieval with heat in high $\mathrm{pH}$ buffer on the BondMax Autostainer (Vision BioSystems Ltd) using the Bond Polymer Define Detection System and using 3,3'-diaminobenzidine as the chromogen (Vision Biosystems Ltd) to detect antigen expression.

\section{Immunohistological scoring}

The areas of highest protein expression evident at low-power scanning were considered for analysis. Staining was scored as negative only after careful examination of the entire tissue section under high magnification $(\times 1,000)$. Quantification of the number of positive tumor cells was performed simultaneously by two investigators (AC and IV) with no previous knowledge of clinical outcome or other clinical variables. A Nikon microscope (model Eclipse E200) was used to score at least 500 cells in high-power fields. We considered CD95 expression as positive in cells with nuclear, membrane, or cytoplasmic staining. P53 and BCL2 expression were considered as positive in cells with nuclear staining and cytoplasmic staining, respectively. Specimens that contained $>10 \%$ positive tumor cells for each marker were scored as positive; those with $\leq 10 \%$ were scored as negative.

Statistical analysis

Statistical analysis was performed using the SPSS 15.0 software program. We performed the Kaplan-Meier survival analysis to compare patients with positive and negative immunohistochemical results for each marker. $p<0.05$ was used to indicate statistical significance. Comparison between survival curves was done by using the log-rank test. We used Cox-regression analysis to estimate the odds ratio with a $95 \%$ confidence interval in order to determine independent prognostic factors for survival.

\section{Results}

Characteristics of the patient population

All patients had been staged according to the Ann Arbor staging system. Twenty six patients $(36.6 \%)$ had been classified as stage I, $19(26.7 \%)$ as stage II, $10(14.1 \%)$ as stage III, and $16(22.6 \%)$ as stage IV. Elevated levels of serum lactate dehydrogenase had been observed in 40 patients $(56.3 \%)$. Eight patients $(11.3 \%)$ exhibited an IPI score of 0,18 (25.3\%); an IPI score of $1,17(23.9 \%)$; an IPI score of 2, $15(21.1 \%)$; an IPI score of 3,11 (15.5\%); an IPI score of 4 and $2(2.9 \%)$; an IPI score of 5 . The patients' clinical characteristics are summarized in Table 2.

Overall, 26 patients $(36.6 \%)$ exhibited low (L) IPI score (0-1 risk factors), 17 (23.9\%) low/intermediate (LI) IPI score (2 risk factors), 15 (21.1\%) high/intermediate (HI) IPI score (3 risk factors) and 13 (18.4\%) high $(\mathrm{H})$ IPI score (4-5 risk factors). The 2-year survival rate for the patients with L, LI, HI and H IPI scores was $80.8 \%$, $58.8 \%, 53.3 \%$ and $38.4 \%$, respectively. The 5-year survival rate was $69.2 \%$ for patients with L IPI score, $47.1 \%$ for LI IPI score, $46.6 \%$ for HI IPI score and $23.1 \%$ for H IPI score. The 2-year and 5-year survival rates are presented in Table 3.

Table 2 Characteristics of the patients with extranodal NHLs $(N=71)$

\begin{tabular}{lc}
\hline Median age (range), years & $65.8(16-83)$ \\
\hline Sex (\%) & $35(49.2)$ \\
Female & $36(50.8)$ \\
Male & \\
Stage (\%) & $26(36.6)$ \\
I & $19(26.7)$ \\
II & $10(14.1)$ \\
III & $16(22.6)$ \\
IV & \\
Symptoms (\%) & $44 / 27(61.9 / 38.1)$ \\
A/B & \\
LDH levels (\%) & $31 / 40(43.7 / 56.3)$ \\
Normal/elevated & \\
IPI score (\%) & $18(25.3)$ \\
0 & $17(23.9)$ \\
1 & $15(21.1)$ \\
2 & $11(15.5)$ \\
3 & $2(2.9)$ \\
4 & \\
5 &
\end{tabular}

LDH lactate dehydrogenase, IPI International Prognostic Index 
Table 3 Two-year and 5-year survival rates according to IPI score

\begin{tabular}{lrlrcrc}
\hline IPI & RF & \multicolumn{2}{c}{ Patients (\%) } & Patients 2 years (\%) & Patients 5 years (\%) \\
\hline L & $0-1$ & 26 & 36.6 & 21 & 80.8 & 18 \\
LI & 2 & 17 & 23.9 & 10 & 58.8 & 89.2 \\
HI & 3 & 15 & 21.1 & 8 & 53.3 & 77.1 \\
H & $4-5$ & 13 & 18.4 & 5 & 38.4 & 3 \\
\hline
\end{tabular}

IPI International Prognostic Index, $L$ low, $L I$ low/intermediate, $H I$ high/intermediate, $H$ high, $R F$ risk factors

\section{Expression of CD95, P53, and BCL2}

We studied the expression of CD95, P53, and BCL2 in DLBCL and MALTL.

\section{$D L B C L(N=45)$}

Twenty-two cases (48.9\%) showed positive staining ( $>10 \%$ of tumor cells) for CD95; negative cases (staining in $\leq 10 \%$ of tumor cells) showed only scattered positive cells. CD95 expression proved to be a statistically significant favorable prognostic factor in predicting survival. The 5-year overall survival rate for CD95-positive and CD95-negative groups was $71.5 \%$ and $35 \%$, respectively ( $p=0.004$; Fig. 1a).

Twenty-four $(53.3 \%)$ cases showed positive staining $(>10 \%$ of tumor cells) for BCL2 and negative cases (staining in $\leq 10 \%$ of tumor cells) showed only scattered positive cells. BCL2 expression was found to be a statistically significant unfavorable prognostic factor in predicting survival. The 5-year overall survival rate was $37 \%$ for the BCL2-positive cases and $70.8 \%$ for the BCL2negative cases ( $p=0.009$; Fig. 1c).

There was no significant difference in the overall survival between P53-positive and P53-negative groups ( $p=0.076$; Fig. 1b).

Multivariate models that included International Prognostic Index score, CD95, and BCL2 expression showed that IPI score $(p=0.011)$, positive expression of CD95 $(p=0.024)$, and negative expression of BCL2 ( $p=0.032)$ were independent prognostic factors for overall survival.

\section{$\operatorname{MALTL}(N=26)$}

Nineteen cases $(73 \%)$ were positive $(>10 \%$ of tumor cells) for CD95. Negative cases (staining in $\leq 10 \%$ of tumor cells) showed only scattered positive cells. CD95 expression seemed to be a statistically significant favorable prognostic factor in predicting survival. The 5 -year survival rate was significantly better in CD95-positive cases $(89.5 \%)$ when compared to CD95-negative cases $(42.9 \% ; p=0.004$; Fig. 2a).

Eight cases $(30.7 \%)$ showed positive staining ( $>10 \%$ of tumor cells) for P53; negative cases (staining in $\leq 10 \%$ of tumor cells) showed only scattered positive cells. P53 expression proved to be a statistically significant unfavorable prognostic factor in predicting survival. The 5-year overall survival rate for the P53-negative and P53-positive groups was $88.9 \%$ and $50 \%$, respectively ( $p=0.016$; Fig. $2 b$ ).

There was no significant difference in the overall survival between BCL2-positive and BCL2-negative groups ( $p=0.080$; Fig $2 \mathrm{C}$ ).

CD95 and P53 expression (positive vs. negative result) and International Prognostic Index Score, were statistically analyzed using Cox regression analysis. The results showed that IPI score $(p=0.009)$, positive expression of CD95 $(p=$ $0.016)$ and negative expression of P53 $(p=0.035)$ were independent prognostic factors for overall survival.

\section{Discussion}

Extranodal non-Hodgkin's lymphomas have heterogeneous clinical features and vary markedly in response to treatment and prognosis. It is important to identify causes that could be responsible for the failure or success of chemotherapy in patients with similar pathological and clinical characteristics.

In our study, we investigated the relationship between the expression of CD95, BCL2 (both regulators of apoptosis), P53 (cell cycle control protein) in extranodal DLBCLs and MALTLs in relation to prognosis. This is the first paper in our best knowledge and after extensive review of the literature that examines the relationship of these proteins with survival in patients with extranodal DLBCLs and MALTLs.

Loss of CD95 (FAS) gene function has been proposed as an important step in early MALT lymphoma development and CD95 mutations have been recognized in malignant lymphomas, particularly at extranodal sites. Primary gastric DLBCLs frequently exhibit resistance to CD95-mediated apoptosis, suggesting that these mutations contribute to the pathogenesis of gastric lymphomas by rendering lymphocytes resistant to apoptosis [13]. Many studies suggested that lymphomas with high CD95 protein expression tend to be more sensitive to chemotherapy than those with low expression levels [13]. 
Fig. 1 a-c Overall survival of patients with DLBCL in relation to CD95 (a), P53 (b), and BCL2 (c) expression

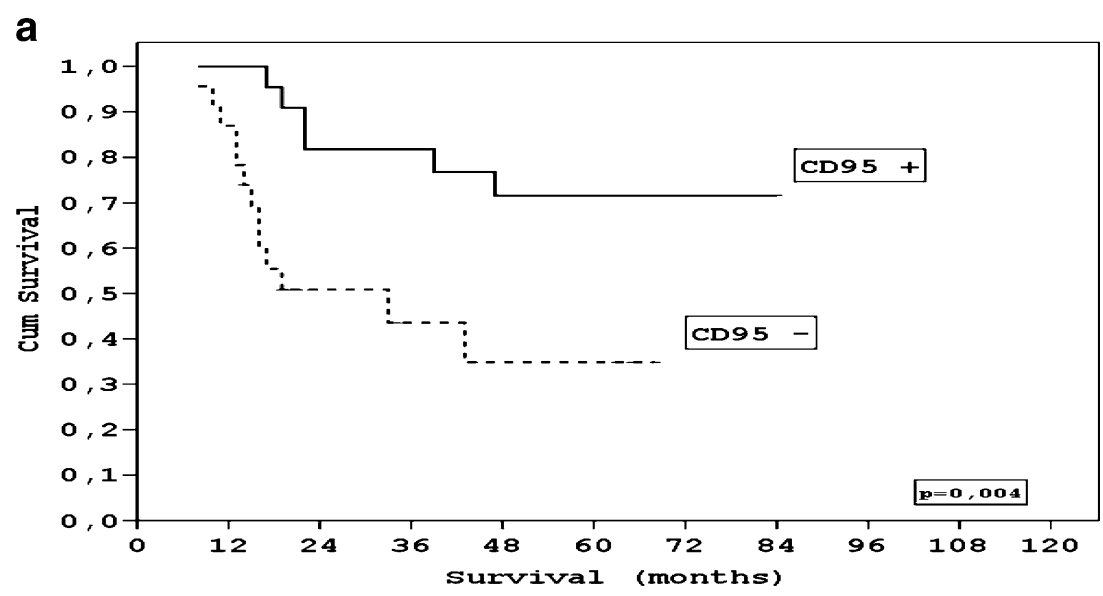

b

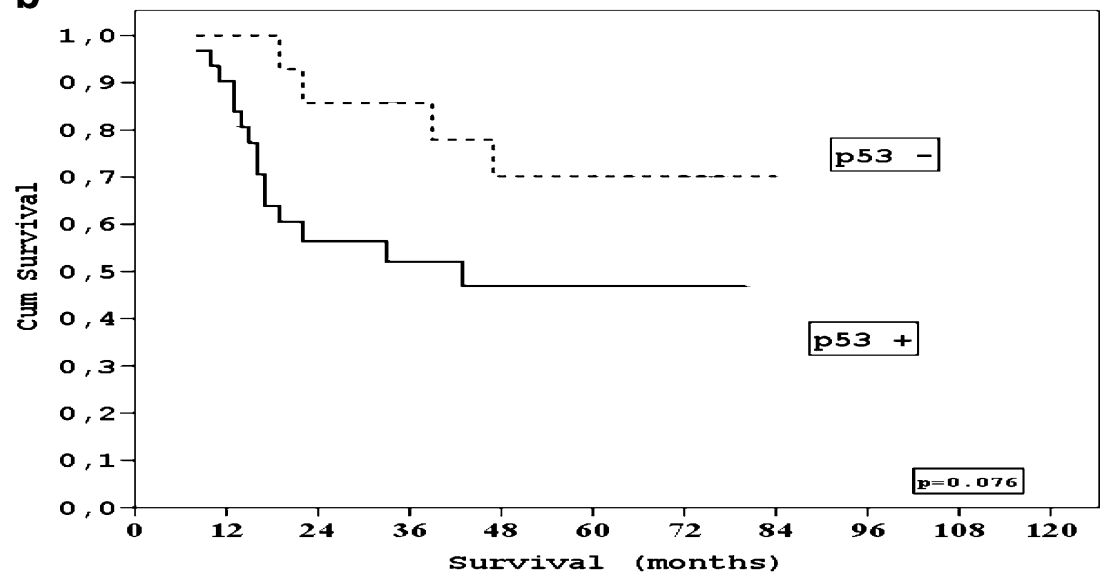

C

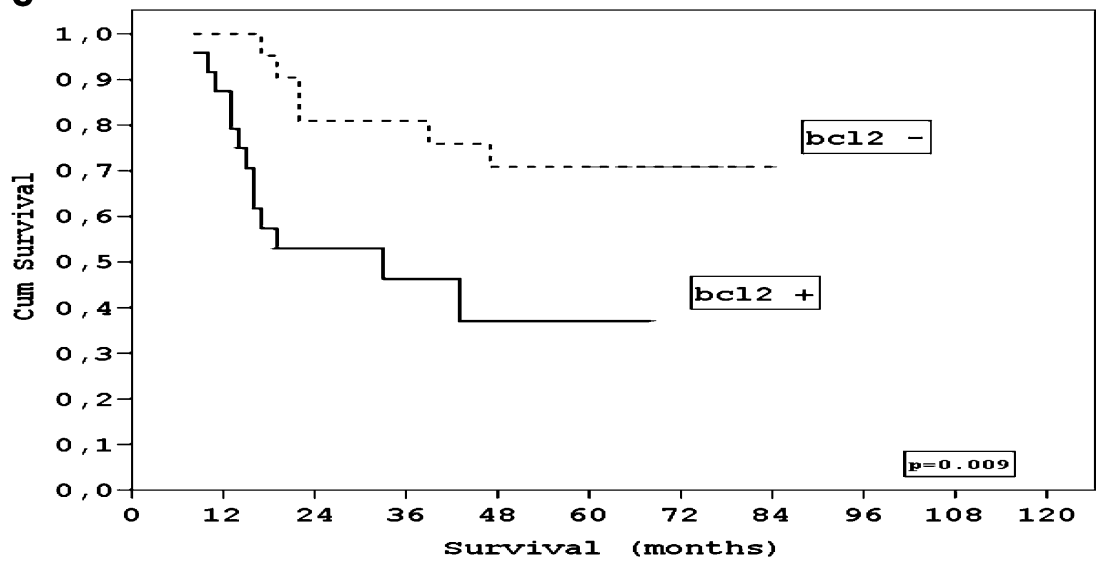

In our study, we concluded that patients with CD95 positive expression had better survival than those with negative expression in extranodal DLBCLs and MALTLs.

Mutations in P53 tumor suppressor gene are the most common genetic lesions observed in human malignant tumors and occur moderately often in hematopoietic malignancies. They are particularly associated with progression of disease in both lymphoid and myeloid leukemias as well as lymphomas. P53 mutations occur very frequently in Burkitt's lymphoma and are observed in about $30 \%$ of aggressive B-cell lymphoma cases [14]. A previous study reported that shorter survival of patients with lymphomas with P53 protein overexpression was due to increased drug resistance. This finding was independent of other parameters such as tumor mass, performance status, histological grade and stage of the disease [15]. Another study demonstrated that wild-type P53 protein status is a necessary prerequisite for CD95 expression and for a functional downstream CD95-mediated apoptotic pathway [16]. This knowledge resulted in research efforts 
Fig. 2 a-c Survival curves for patients with MALTLs with negative and positive CD95 expression (a), P53 (b), and BCL2 (c)

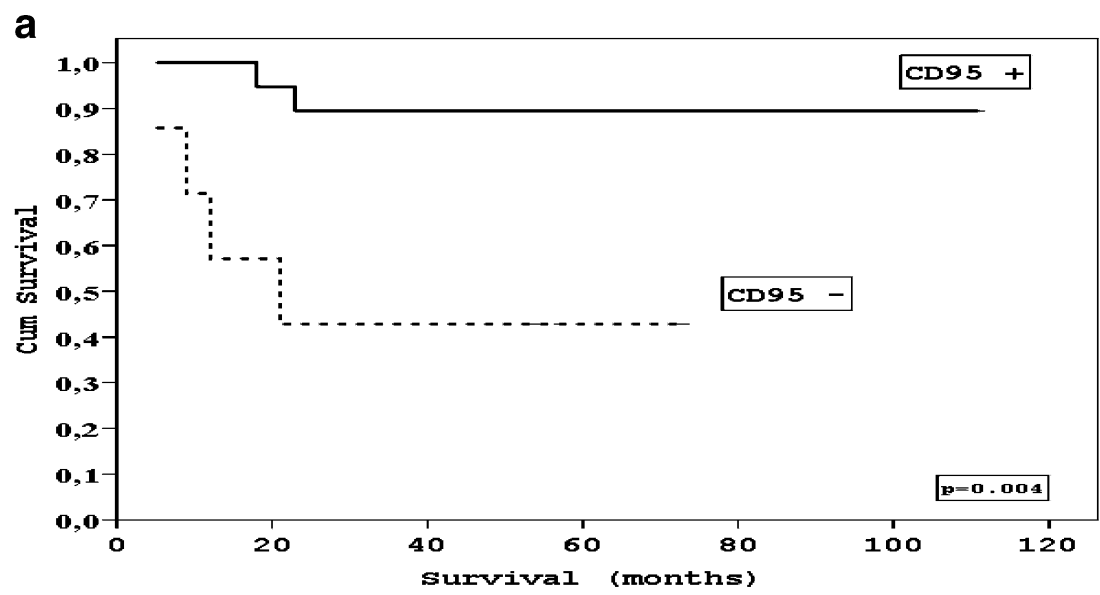

b

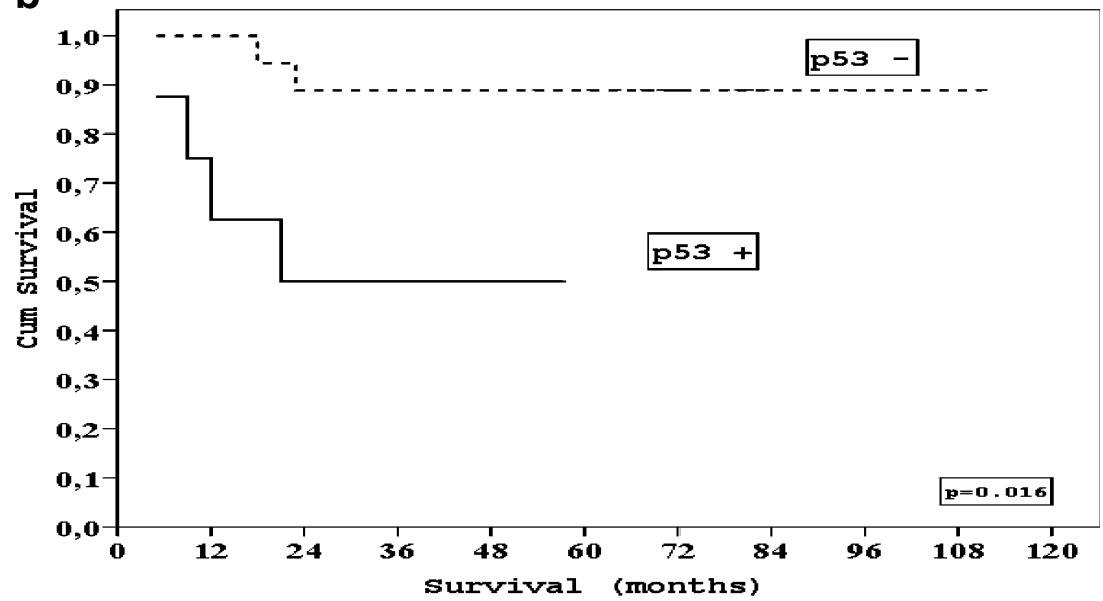

C

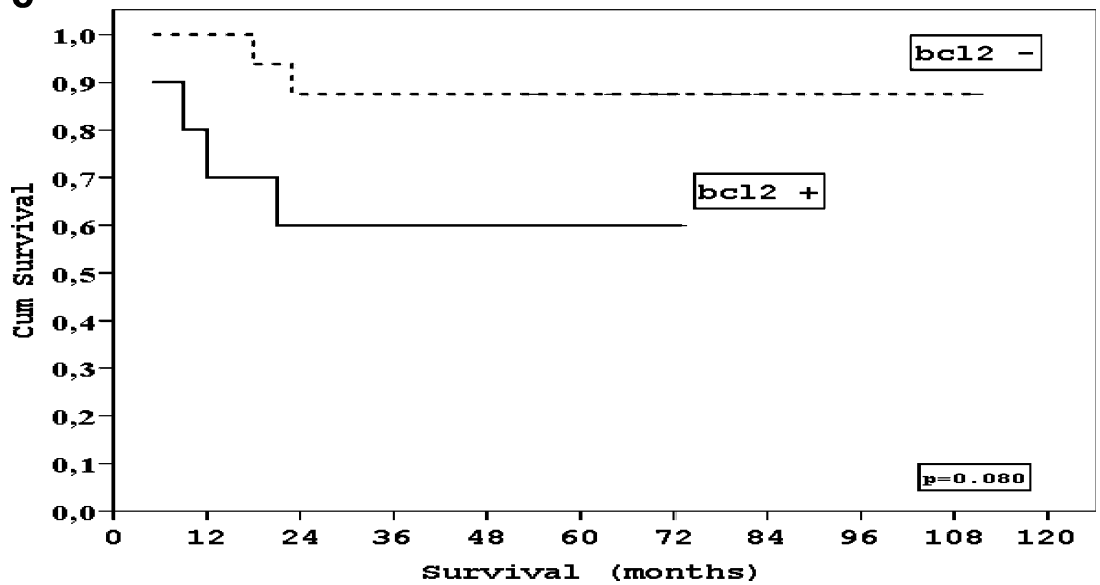

in order to clarify the specific interactions between wild-type P53 protein and the CD95 signal transduction pathway in human cancer cell lines [17]. It is possible to test novel anticancer drugs for their ability to induce CD95-mediated apoptosis on these cancer cell lines and also to determine the cytotoxicity effects of anticancer agents in relation to P53 status [18, 19]. Piris et al [20] suggested that simultaneous expression of BCL2 and P53 proteins was associated with a poorer prognosis than expression of P53 alone.

In our study, we report a statistically significant negative correlation between P53 expression and survival in patients with MALTLs. In extranodal DLBCLs, there was no significant difference in the overall survival between P53positive and P53-negative groups. Nevertheless, there was a 
clear trend showing that patients with positive expression of P53 died sooner than patients with P53-negative lymphomas.

$B C L 2$ is an anti-apoptotic gene that is found at inappropriately high levels in probably $>50 \%$ of all cancers [21]. The role of BCL2 in chemotherapy-induced apoptosis is very important [22]. BCL2-positive cells are resistant to chemotherapeutic agents since the drugs are unable to kill tumor cells by inducing apoptosis [23]. Overexpression of the BCL2 protooncogene has been reported to inhibit the apoptosis mediated by the CD95 antigen [24]. Various studies have shown an inverse association between CD95 and BCL2 expression in normal germinal center cells and normal peripheral blood lymphocytes, monocytes, and neutrophils. However, the relationship of these two proteins in lymphoid neoplasms appears to be less well defined. Since BCL2 expression may provide protection against apoptosis for tumors that are CD95-positive, the observation of frequent co-expression of BCL2 and CD95 in low-grade B-cell lymphomas may explain the relative longevity of these neoplastic cells [25]. Nguyen et al [26] suggested that in contrast to low-grade lymphomas where the BCL2 protein is more often co-expressed with CD95, two-thirds of high-grade CD95-positive B-cell lymphomas lack BCL2 expression. This finding raises the possibility that in these high-grade lymphomas, other regulators besides BCL2 may be involved in the modulation of the apoptosis signal mediated by CD95. In any case, it is observed that these CD95+/BCL2- high-grade lymphomas follow a less aggressive course compared to their CD95-/ BCL2- counterparts [26].

In our study, we found that in patients with extranodal DLBCLs, the overall survival was lower in BCL2-positive cases compared to negative ones. Therefore, patients with BCL2 positive extranodal DLBCLs should probably receive a more intensive or even a modified chemotherapeutic treatment. We did not find any statistically important prognostic significance of BCL2 expression in MALTLs; however, patients with BCL2-negative MALTLs seemed to survive longer than those with positive expression.

In conclusion, in our study CD95 protein expression appeared to be a favorable independent prognostic factor in patients with extranodal DLBCLs and MALTLs, negative expression of P53 protein was found to be an independent unfavorable prognostic factor of overall survival in MALTLs, and BCL2 protein expression was found to be unfavorably associated with survival in extranodal DLBCLs.

Acknowledgments The authors gratefully acknowledge the collaboration of the technical staff of the Pathology Department.

Authorship and disclosures $\mathrm{AC}$ was the principal investigator and analyzed the data. IV and NP participated in the scoring of immunohistochemical results. GT and GA participated in the statistical analysis.

\section{References}

1. Feller AC, Diebold J (2004) Histopathology of nodal and extranodal non-Hodgkin's lymphomas. 3rd, completely rev. and updated ed. Springer, Berlin, p 186

2. Irisarri M, Plumas J, Bonnefoix T et al (2000) Resistance to CD95mediated apoptosis through constitutive c-FLIP expression in a nonHodgkin's lymphoma B cell line. Leukemia 14:2149-2158

3. Troeger A, Glouchkova L, Escherich G et al (2008) Reduced expression of defective modulation of TNF receptor/ligand family molecules on proB-ALL blasts. Klin Pediatr 220:353-357

4. Griffith TS, Yu X, Herndon JM, Green DR, Ferguson TA (1996) CD95-induced apoptosis of lymphocytes in an immune-privileged cite induces immunological tolerance. Immunity 5:7-16

5. Landowski TH, Moscinski L, Burke R (2001) CD95 antigen mutations in hematopoietic malignancies (review). Leuk Lymphoma 42:835-846

6. Wilson WH, Teruya-Feldstein J, Fest T (1997) Relationship of P53, BCL2, and tumor proliferation to clinical drug resistance in non-Hodgkin's lymphomas. Blood 89:601-609

7. Harris SL, Levine AJ (2005) The P53 pathway: positive and negative feedback loops (review). Oncogene 24:2899-2908

8. Steele RJ, Lane DP (2005) P53 in cancer: a paradigm for modern management of cancer (review). Surg 3:197-205

9. Kroemer G (1997) The proto-oncogene BCL2 and its role in regulating apoptosis. Nat Med 3:614-620

10. Chao DT, Korsmeyer SJ (1998) BCL-2 family: regulators of cell death. Annu Rev Immunol 16:395-419

11. Pazella F, Turley H, Kuzu I, Tungekar MF, Dunnill MS, Pierce CB et al (1993) BCL2 protein in non-small-cell lung carcinoma. N Engl J Med 329:690-694

12. Pazella F, Gatter K (1995) What is the value of BCL2 protein detection for histopathologists? Histopathology 26:89-93

13. Wohlfart S, Sebinger D, Gruber P (2004) FAS (CD95) mutations are rare in gastric MALT lymphoma but occur more frequently in primary gastric diffuse large B-cell lymphomas DLBCL. Am J Pathol 164:1081-1089

14. Imamura J, Miyoshi I, Koeffler HP (1994) P53 in hematologic malignancies. Blood 84:2412-2421

15. Hernandez L, Fest T, Cazorla M et al (1996) P53 gene mutations and protein overexpression are associated with aggressive variants of mantle cell lymphomas. Blood 87:3351-3359

16. Li Y, Raffo AJ, Drew L, Mao Y (2003) FAS-mediated apoptosis is dependent on wild-type P53 status in human cancer cells expressing a temperature-sensitive P53 mutant alanine-143. Cancer Res 63:1527-1533

17. Das GC, Holiday D, Gallardo R, Haas C (2001) Taxol-induced cell cycle arrest and apoptosis: dose-response relationship in lung cancer cells of different wild-type P53 status and under isogenic condition. Cancer Lett 165:147-153

18. Stewart ZA, Tang LJ, Pietenpol JA (2001) Increased P53 phosphorylation after microtubule disruption is mediated in a microtubule inhibitor- and cell-specific manner. Oncogene 20:113-124

19. Makin G, Hickman JA (2000) Apoptosis and cancer chemotherapy. Cell Tissue Res 301:143-152

20. Piris MA, Pezella F, Martinez-Montero JC et al (1994) P53 and BCL2 expression in high-grade B-cell lymphomas: correlation with survival time. Br J Cancer 69:337-341

21. Reed JC (1995) Regulation of apoptosis by BCL2 family proteins and its role in cancer and chemoresistance. Curr Opin Oncol 7:541-546

22. Hermine O, Haioun C, Lepage E (1996) Prognostic significance of BCL2 protein expression in aggressive non-Hodgkin's lymphoma. Groupe d'Etude des Lymphomes de l'Adulte (GELA). Blood 87:265-272 
23. Miyashita T, Reed JC (1993) BCL2 oncoprotein blocks chemotherapyinduced apoptosis in a human leukemia cell line. Blood $81: 151-157$

24. Iwai K, Miyawaki T, Takizawa T (1994) Differential expression of BCL2 and susceptibility to anti-Fas-mediated cell death in peripheral blood lymphocytes, monocytes, and neutrophils. Blood 84:1201-1208
25. Yoshino T, Kondo E, Cao L (1994) Inverse expression of BCL2 protein and FAS antigen in lympho-blasts in peripheral blood $\mathrm{T}$ and B lymphocytes. Blood 83:1856-1861

26. Nguyen PL, Harris NL, Ritz J, Robertson MJ (1996) Expression of CD95 antigen and BCL2 protein in non Hodgkin's lymphomas and Hodgkin's disease. Am J Pathol 148:847-853 